\title{
Histological Analysis of Reproductive System in Low-Dose Nonylphenol- treated F1 Female Mice
}

\author{
Yong-Bin Kim ${ }^{1}$, Yong-Pil Cheon ${ }^{2}$, Donchan $\mathrm{Choi}^{3}$, and ${ }^{\dagger}$ Sung-Ho Lee ${ }^{1}$ \\ 'Dept. of Biotechnology, Sangmyung University, Seoul 03016, Korea \\ ${ }^{2}$ Division of Developmental Biology and Physiology, School of Biological Sciences and Chemistry, Sungshin \\ University, Seoul 02844, Korea \\ ${ }^{3}$ Dept. of Life Science, College of Environmental Sciences, Yong-In University, Yongin 17092, Korea
}

\begin{abstract}
Previously, we reported adverse effects of low-dose nonylphenol (NP) exposure on the reproductive parameters of $\mathrm{F} 1$ female mice. In the present study we further investigated the pathohistological effect of NP exposure on the reproductive organs in F1 female mice. NP exposures were continuously conducted from parental pre-mating period until the postnatal day (PND) 33 of F1 offspring for vaginal examination. Mice were sacrificed on PND 30 and the reproductive tissue weights were measured. The initial (at PND 21) body weights of the NP-50 group animals were significantly lower than those of control group animals, and the weight deficit were recovered when the terminal (PND 33) body weights were measured. Early vaginal opening was found in NP group animals $(p<0.05)$. Pathohistological studies revealed that NP-treated F1 animals showed prominent increase in the ovarian follicle numbers $(p<0.01)$, and decrease in the diameter of uterine myometrium $(p<0.01)$, and increase in the diameter of luminal epithelium $(p<0.05)$. The present study demonstrated that the subchronic low-dose NP exposure induced early beginning of puberty and pathohistological abnormalities in ovary and uterus of F1 mice. Further studies are needed to achieve a better understanding on the action mechanism of NP in pubertal onset and to find a way to avoid a hazardous situation provoked by NP exposure.
\end{abstract}

Keywords: Nonylphenol, F1 female mice, Ovary, Uterus, Histology

\section{INTRODUCTION}

It is evident that nonylphenol (NP), a commonly used industrial chemical, is an endocrine disrupting chemical (EDC) with estrogenic activity exerting adverse effects on health (Soto et al., 1991; Noorimotlagh et al., 2017; Rattan et al., 2018). In rodents studies, ample evidence has proven that NP could interfere with reproductive system (Nagao et al., 2001; Han et al., 2004; Gong et al., 2009; Mehranjani et al., 2009; Uguz et al., 2009; Aly et al., 2012; Lu et al., 2014). In addition, we also demonstrated that the subchronic low-dose NP exposure resulted in adverse effects on the reproductive parameters of male and female F1 mice, such as decreased weight of ovary and uterus in F0 animals, decreased rate of ovulation in female F1 offspring, increased weight of the testis, decreased weights of the epididymis, prostate and seminal vesicle in male F1 offspring (Cha et al., 2017; Kim et al., 2019). 
Ethics approval

All the animals received humane care in accordance with the guides for animal experiments of the Association for Assessment and Accreditation of Laboratory Animal Care at Sangmyung University (approval number R-1601-1).
In the present study, we used the identical subchronic low-dose NP exposure model to clarify the potent pathohistological effect of NP exposure on the reproductive organs in F1 female mice.

\section{MATERIALS AND METHODS}

\section{Animals}

ICR mice were provided by DBL (Chungcheongbuk-do, Korea) and reared in Sangmyung University animal facility under photoperiods of $12 \mathrm{~h} \mathrm{light/dark} \mathrm{with} \mathrm{lights} \mathrm{on} \mathrm{at} 7 \mathrm{AM}$ and constant temperature of $21^{\circ} \mathrm{C}-23^{\circ} \mathrm{C}$. Food and tap water were supplied ad libitum. The animal protocols were approved by the Animal Care and Use Committees at Sangmyung University (approval number R-1601-1). All the animals received humane care in accordance with the guides for animal experiments of the Association for Assessment and Accreditation of Laboratory Animal Care (AAALAC).

\section{Treatments and tissue preparations}

After acclimation (2 weeks), breeding pairs of mice were divided into 2 groups; (1) Control (CTL), supplied tap water, and (2) NP-50, treated with low-dose NP (50 $\mu \mathrm{g} / \mathrm{L}$; Sigma-Aldrich, St. Louis, MO, USA) via drinking water. The parental $(\mathrm{P})$ generation mice had pre-mating period for 2 weeks. After pre-mating period, male and female mice were placed in the breeding cage until a vaginal plug of female mice was observed. The animals were treated with NP from pre-mating period to weaning of offspring (F1). The F1 generation mice were weaned at postnatal day (PND) 21 and consistently treated with the NP until sacrifice at PND 30. The dates of vaginal opening (VO) were measured until PND 33. Animals were sacrificed, and sample collections were done between 18:00-19:00 pm. The tissues were fixed in paraformaldehyde (4\%) for histological study.

\section{Paraffin tissue section}

Fixed tissues (ovaries and uteri) were dehydrated in graded concentrations of ethanol (70\%, $80 \%, 90 \%, 95 \%$, and 100\%; Duksan, Korea) for 1 hour 30 minutes in each with gentle shaking and soaked in absolute ethanol overnight. The tissues were immersed in xylene (Samchun Chemical, Seoul, Korea) for 30 minutes, 3 times and in paraffin at $56^{\circ} \mathrm{C}$ for 30 minutes, 3 times. The tissues were embedded in paraffin and sectioned (Microm, Walldorf, Germany) at $5 \mu \mathrm{m}$. The samples were attached on microscope slides and the slides were stained with hematoxylin (Sigma-Aldrich) and eosin (Across, USA) for 5 minutes, respectively.

\section{Statistical analysis}

Values were expressed as mean $\pm \mathrm{SE}$ Data were analyzed using Student's $t$-test as indicated. $p<0.05$ was considered statistically significant. Calculations were performed using Graphpad Software Prism version 6.

\section{RESULTS}

\section{Changes in body weights}

To find the effect of subchronic low-dose exposure to NP on the weight gain of F1 female mice, we measured and compared the body weights at weaning day (PND 21) and at sacrifice day (PND 30) (Table 1; $n=9)$. The initial body weights of the NP-50 group animals were significantly 
Table 1. Effect of subchronic low-dose exposure to NP on the body weight gain from PND 21 to PND 30 in $\mathrm{F} 1$ female mice

\begin{tabular}{lcc}
\hline \hline & CON & NP-50 \\
\hline Initial body weight $(\mathrm{g})$ & $10.6 \pm 0.2$ & $9.0 \pm 0.2^{*+* *}$ \\
Terminal body weight $(\mathrm{g})$ & $23.2 \pm 0.4$ & $23.2 \pm 0.3$ \\
Increase rate of body weight $(\%)$ & $218 \pm 3$ & $259 \pm 5^{* * *}$ \\
\hline
\end{tabular}

Values are expressed as mean $\pm S E(n=9)$.

$p<0.001$, significantly different from the control group.

NP, nonylphenol; PND, postnatal day; CON, control group provided with tap water only; NP-50, treated with nonylphenol at a dose of $50 \mu \mathrm{g} / \mathrm{L}$.

lower than those of control group animals $(p<0.001)$. However, the terminal body weights of the NP-50 group animals were not significantly different than those of control animals. Consequently, the increment rate of body weight in NP-50 group animals were significantly lower than those in control group animals $(p<0.001)$.

\section{Changes in VO index}

Fig. 1 indicates that advanced VO was occurred in NP-50 group animals compared to those in control animals. The average date of $\mathrm{VO}$ in control and NP-50 group is $28.9 \pm 0.89$ and $26.5 \pm 0.52$, respectively $(p<0.05, \mathrm{n}=9)$.

\section{Pathohistological changes in ovary and uterus}

The overall size and shape of the NP-50 group ovaries were relatively bigger than control ovaries (Fig. 2). No corpus luteum (CL) was found in the control ovaries while several CL were present in NP-50 ovaries. Likewise, the primary and secondary follicles were more abundant in NP-50 ovaries

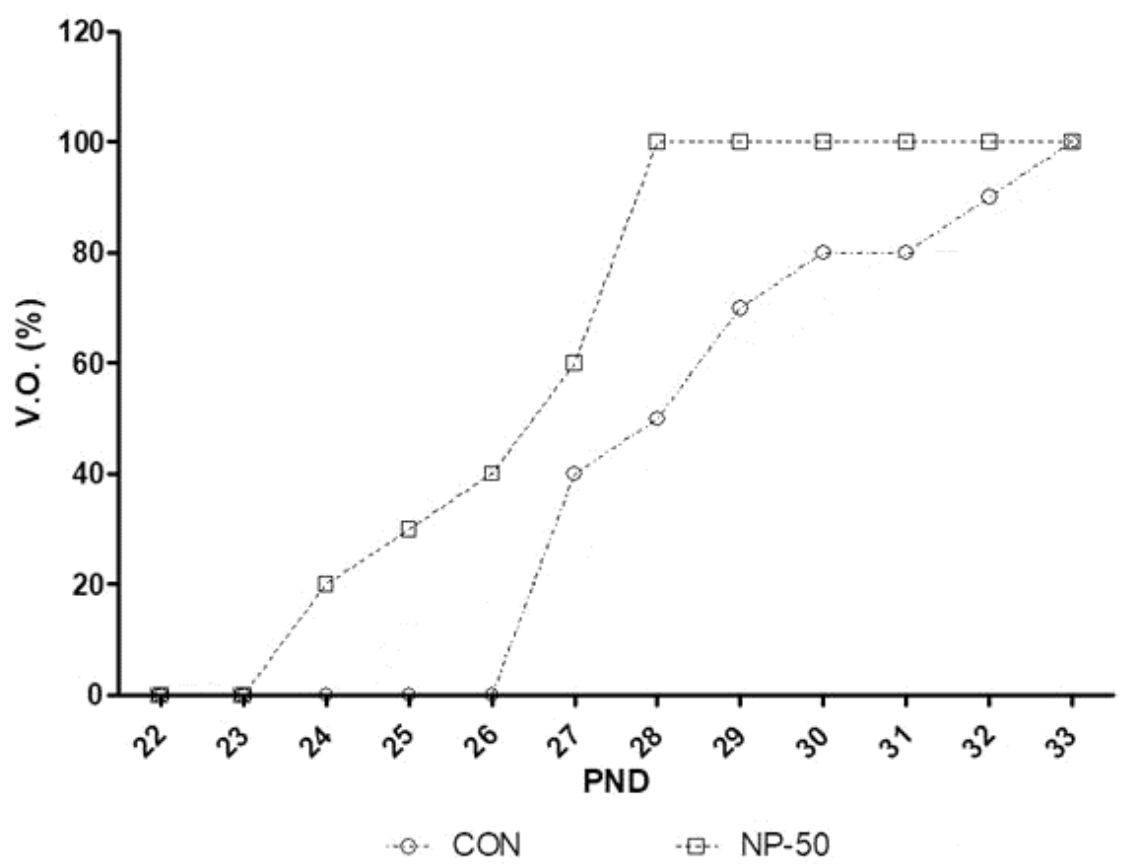

Fig. 1. Dates of vaginal opening in F1 female mice treated with NP. Expressed as a percentage of total number of animals per experimental group. Values are expressed as mean $\pm S E(n=9)$. NP, nonylphenol; CON, control group provided with tap water only; NP-50, treated with nonylphenol at a dose of $50 \mu \mathrm{g} / \mathrm{L}$; V.O., vaginal opening. 


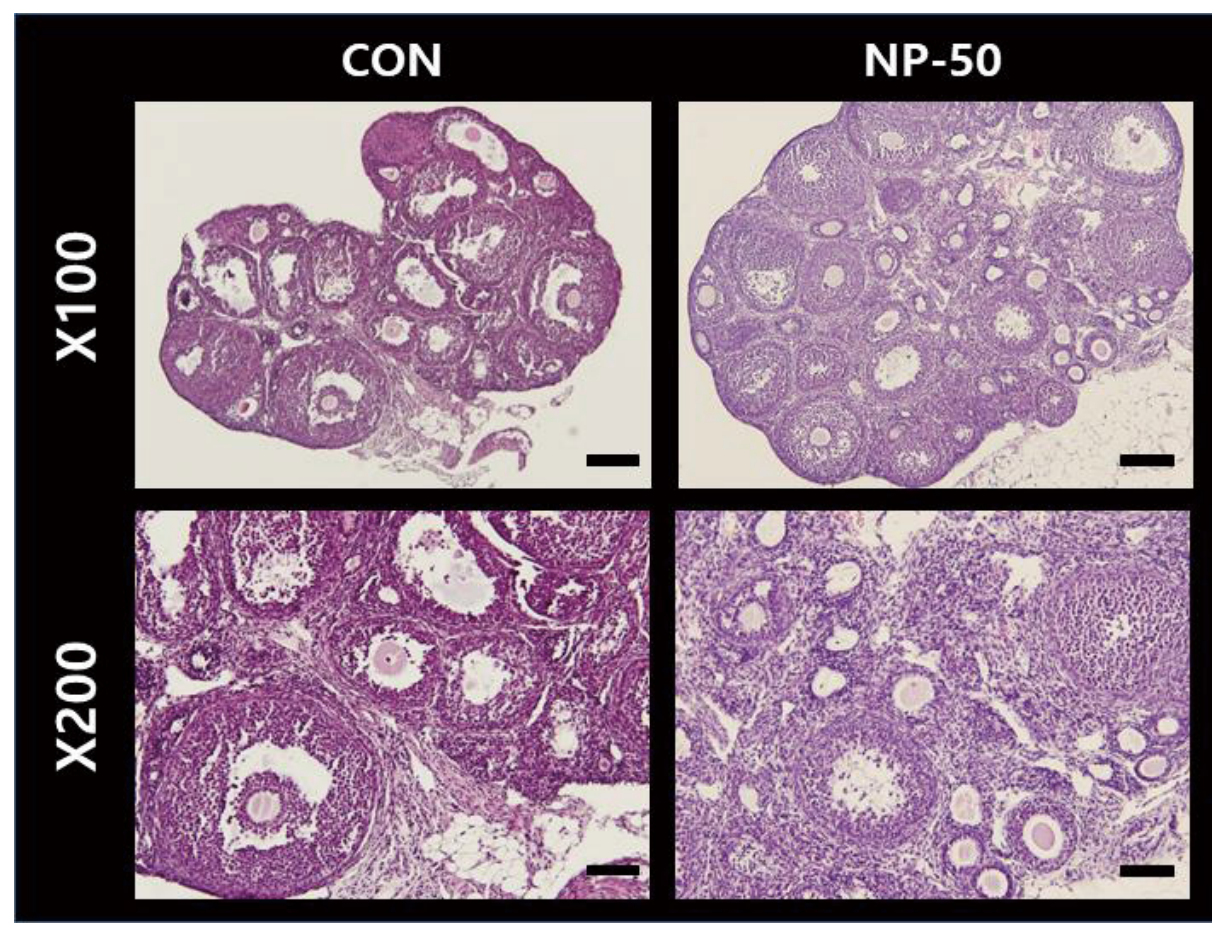

Fig. 2. Microphotographs of the ovaries in F1 mice (PND 30). The paraffin-embedded tissues were sectioned at $5 \mu \mathrm{m}$ and the sections were stained with hematoxylin and eosin. Note that multiple corpora lutea and abundant primary and secondary follicles were found in NP-50 ovaries. Scale bar $\times 100=80 \mu \mathrm{m}$, $\times 200=40 \mu \mathrm{m}$. CON, control group provided with tap water only; NP-50, treated with nonylphenol at a dose of $50 \mu \mathrm{g} / \mathrm{L} ; \mathrm{NP}$, nonylphenol; PND, postnatal day.

(Table 2; $\mathrm{n}=4$ ). The overall size and shape of the NP-50 group uteri were relatively smaller than those of control uteri (Fig. 3). Prominently thin layers of endometrium and myometrium, thick luminal epithelia, well-developed luminal structure and less number of gland were found in NP-50 group uteri (Table $2 ; \mathrm{n}=4)$.

\section{DISCUSSION}

The present study demonstrated that the subchronic low-dose NP exposure induced early beginning of puberty and pathohistological abnormalities in ovary and uterus of $\mathrm{F} 1$ mice. Previously, we demonstrated that the chronic lower-dose NP administration induced significant alterations such as weight of gonads and accessory organs and number of ovulated oocytes in F1

Table 2. Effect of NP on ovarian and uterine histological parameters in F1 mice

\begin{tabular}{lcc}
\hline \hline & CON & NP-50 \\
\hline Number of ovarian follicle & $7.8 \pm 0.48$ & $14.2 \pm 1.36^{* *}$ \\
Number of uterine gland & $8.2 \pm 1.02$ & $6.8 \pm 1.44$ \\
Diameter of myometrium $(\mu \mathrm{m})$ & $27.2 \pm 2.08$ & $15.3 \pm 1.97^{* *}$ \\
Diameter of luminal epithelium $(\mu \mathrm{m})$ & $14.1 \pm 1.63$ & $10.9 \pm 0.94^{*}$ \\
\hline
\end{tabular}

Values are expressed as mean $\pm S E(n=4)$.

" $p<0.05, " p<0.01$, significantly different from the control group.

$\mathrm{NP}$, nonylphenol; CON, control group provided with tap water only; NP-50, treated with nonylphenol at a dose of $50 \mu \mathrm{g} / \mathrm{L}$. 


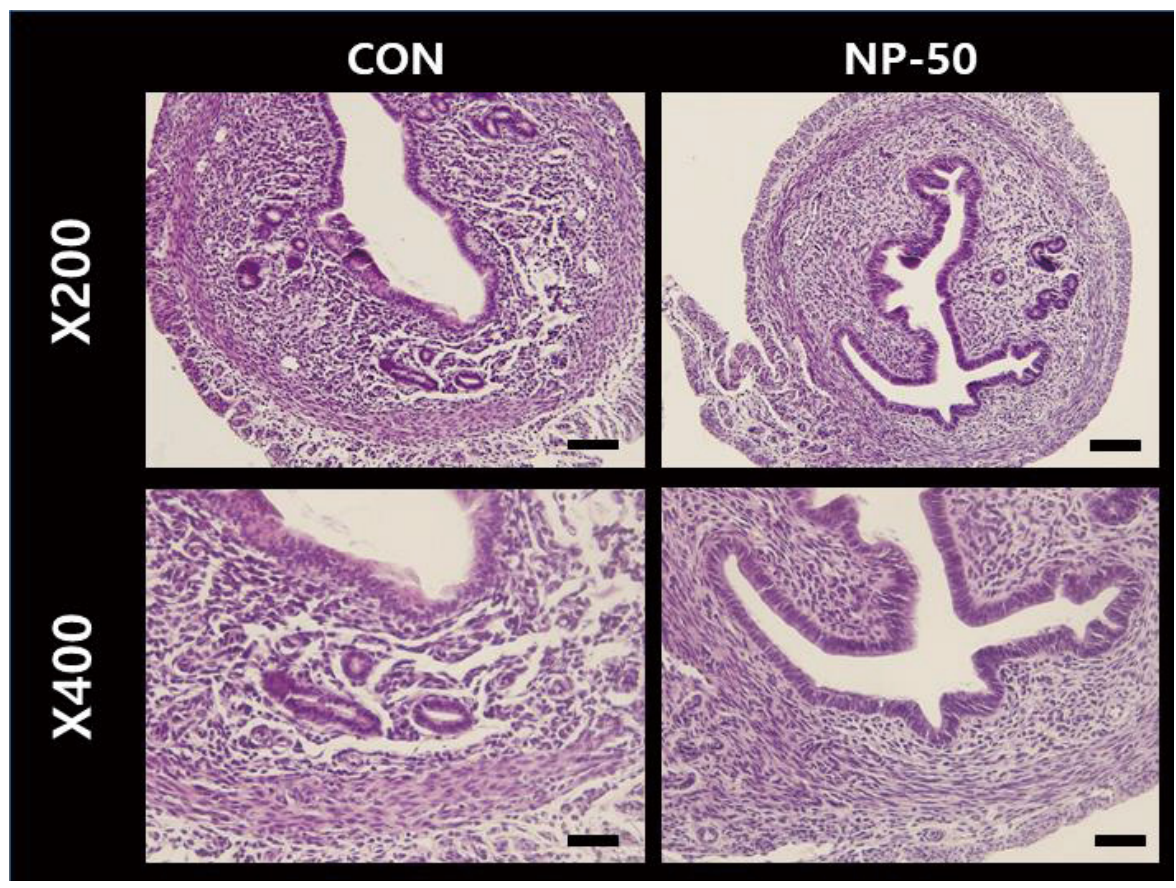

Fig. 3. Microphotographs of the uteri in F1 mice (PND 30). The paraffin-embedded tissues were sectioned at $5 \mu \mathrm{m}$ and the sections were stained with hematoxylin and eosin. Thin layers of endometrium and myometrium, thick luminal epithelia, well-developed luminal structure and relatively poor developed endometrium were found in NP-50 group uteri. Scale bar $\times 200=40 \mu \mathrm{m}, \times 400=20 \mu \mathrm{m}$. NP, nonylphenol; CON, control group given tap water only; NP-50, treated with NP at a dose of $50 \mu \mathrm{g} / \mathrm{L}$; PND, postnatal day.

offspring (Cha et al., 2017). Interestingly, NP-induced changes were found in the absolute weight of the testis and epididymis but not in the relative weights of these organs (Kim et al., 2019). In the same study, however, histological examinations revealed that NP-treated F1 males exerted some extent of abnormalities; decreased seminiferous tubule diameters, reduced luminal area, and lower number of germ cells, and some sloughing morphologies in the tubules. Therefore, our previous and the present studies suggest that subchronic low-dose NP exposure could bring about reproductive toxicity without noticeable weight changes in gonad and/or accessory sex organs. We referred to this as 'qualitative' changes in reproductive tissues that could be derived from the epigenetic reprogramming by low-dose NP exposure which is frequently experienced in daily living (Kim et al., 2019).

NP can mimic the endogenous hormone $17 \beta$-estradiol, and it competes with the endogenous hormone for binding with the estrogen receptors (Soto et al., 1991). Although the concentration of NP in the environment is decreasing, it is still found at concentrations of $4.1 \mu \mathrm{g} / \mathrm{L}$ in river water and $1 \mathrm{mg} / \mathrm{kg}$ in sediments (Soares et al., 2008). Officially approved NOAELs (No Observed Adverse Effect Levels) and LOAELs (Lowest Observed Adverse Effect Levels) in rat for reproductive toxicity (based on decreases in epididymal sperm density or testicular sperm head counts, increases in estrous cycle length, and decreases in ovarian weights) range from 13 to $19 \mathrm{mg} / \mathrm{kg}-\mathrm{BW} / \mathrm{day}$, and from 43 to $64 \mathrm{mg} / \mathrm{kg}-\mathrm{BW} /$ day, respectively (EPA, 2009). The calculated oral NOAEL for repeated dose for humans was $15 \mathrm{mg} / \mathrm{kg} /$ day based on rat studies (Bontje et al., 2012). The dose employed in our previous and the present studies (18.5-19.8 ug/ $/ \mathrm{kg} /$ day) was far lower than NOAEL in rat and human (Cha et al., 2017; Kim et al., 2019). Indeed, the average daily human intake of NP was calculated to be $7.5 \mathrm{ug} /$ day in Germany and to be about $25.32 \mathrm{ug} /$ day in Taiwan (Guenther et 
al., 2002; Lu et al., 2007). Therefore, we believe the dose used in our studies is much closer to the human daily intake level, and our NP treatment regimen is better to obtain practical information on the adverse effect of NP exposure to human (Kim et al., 2019).

In the present study we found that the initial body weights of the NP-50 group animals were significantly lower than those of control group animals, and the weight deficit were rapidly recovered when the terminal body weights were measured (Table 1). We hypothesized that 1) the low body weight of NP-50 group animals at weaning day could be caused by the adverse effect of NP exposure on the growth of F1 animals during intrauterine and/or lactational period, and 2) the fast catch-up with weight deficit in NP-50 group could be obesogenic effect of NP during prepubertal period. Concerning the weight gaining, there is an environmental obesogene hypothesis that proposes the exposure to endocrine disruptors during developmental "window" contributes to adipogenesis and the development of obesity (Hao et al., 2013). Further studies are needed to understand the opposite NP effects on body weight gain during these two periods. Fast weight gain by NP exposure during prepubertal period might be attributed to rapid accumulation of lipid within adipose, then this can be a driving force of early VO. Supporting this idea, previous studies demonstrated that body weight - or body fat - impacts on pubertal onset in female rats and monkeys (Glass et al., 1979; Boukouvalas et al., 2008; Terasawa et al., 2012).

In conclusion, the present study demonstrated that the subchronic low-dose NP exposure induced early beginning of puberty and pathohistological abnormalities in ovary and uterus of F1 mice. Further studies are needed to achieve a better understanding on the action mechanism of NP in pubertal onset and to find a way to avoid a hazardous situation provoked by NP exposure.

\section{REFERENCES}

Aly HA, Domènech Ò, Banjar ZM (2012) Effect of nonylphenol on male reproduction: Analysis of rat epididymal biochemical markers and antioxidant defense enzymes. Toxicol Appl Pharmacol 261:134-141.

Bontje D, Hermens J, Vermeire T, Damstra T (2012) Integrated Risk Assessment: Nonylphenol Case Study. Report No. WHO/IPCS/IRA/12/04. WHO, Geneva, Switzerland.

Boukouvalas G, Antoniou K, Papalexi E, Kitraki E (2008) Post weaning high fat feeding affects rats' behavior and hypothalamic pituitary adrenal axis at the onset of puberty in a sexually dimorphic manner. Neuroscience 153:373-382.

Cha S, Baek JW, Ji HJ, Choi JH, Kim C, Lee MY, Hwang YJ, Yang E, Lee SH, Jung HI, Cheon YP (2017) Disturbing effects of chronic low-dose 4-nonylphenol exposing on gonadal weight and reproductive outcome over one-generation. Dev Reprod 21:121-130.

EPA. 2009. Screening-Level Hazard Characterization: Alkylphenols Category. U.S. Environmental Protection Agency, Washington, DC.

Glass AR, Dahms WT, Swerdloff RS (1979) Body fat at puberty in rats: Alteration by changes in diet. Pediatr Res 13:7-9.

Gong Y, Wu J, Huang Y, Shen S, Han X (2009) Nonylphenol induces apoptosis in rat testicular Sertoli cells via endoplasmic reticulum stress. Toxicol Lett 186:84-95.

Guenther K, Heinke V, Thiele B, Kleist E, Prast H, Raecker T (2002) Endocrine disrupting nonylphenols are ubiquitous in food. Environ Sci Technol 36:1676-1680.

Han XD, Tu ZG, Gong Y, Shen SN, Wang XY, Kang LN, Hou YY, Chen JX (2004) The toxic effects of nonylphenol on the reproductive system of male rats. Reprod Toxicol 19:215-221.

Hao C, Cheng X, Guo J, Xia H, Ma X (2013) Perinatal exposure to diethyl-hexyl-phthalate 
induces obesity in mice. Front Biosci (Elite Ed) 5:725-733.

Kim YB, Cheon YP, Lee SH (2019) Adverse effect of nonylphenol on the reproductive system in F1 male mice: A subchronic low-dose exposure model. Dev Reprod 23:93-99.

Lu YY, Chen ML, Sung FC, Wang PS, Mao IF (2007) Daily intake of 4-nonylphenol in Taiwanese. Environ Int 33:903-910.

Lu WC, Wang AQ, Chen XL, Yang G, Lin Y, Chen YO, Hong CJ, Tian HL (2014) 90d exposure to nonylphenol has adverse effects on the spermatogenesis and sperm maturation of adult male rats. Biomed Environ Sci 27:907-911.

Mehranjani MS, Noorafshan A, Momeni HR, Abnosi MH, Mahmoodi M, Anvari M, Hoseini SM (2009) Stereological study of the effects of vitamin E on testis structure in rats treated with para-nonylphenol. Asian J Androl 11:508-516.

Nagao T, Wada K, Marumo H, Yoshimura S, Ono H (2001) Reproductive effects of nonylphenol in rats after gavage administration: A two-generation study. Reprod Toxicol 15:293-315.

Noorimotlagh Z, Haghighi NJ, Ahmadimoghadam M, Rahim F (2017) An updated systematic review on the possible effect of nonylphenol on male fertility. Environ Sci Pollut Res Int 24:3298-3314.

Rattan S, Brehm E, Gao L, Niermann S, Flaws JA (2018) Prenatal exposure to di(2-ethylhexyl) phthalate disrupts ovarian function in a transgenerational manner in female mice. Biol Reprod 98:130-145.

Soares A, Guieysse B, Jefferson B, Cartmell E, Lester JN (2008) Nonylphenol in the environment: A critical review on occurrence, fate, toxicity and treatment in wastewaters. Environ Int 34:1033-1049.

Soto AM, Justicia H, Wray JW, Sonnenschein C (1991) p-Nonyl-phenol: An estrogenic xenobiotic released from "modified" polystyrene. Environ Health Perspect 92:167-173.

Terasawa E, Kurian JR, Keen KL, Shiel NA, Colman RJ, Capuano SV (2012) Body weight impact on puberty: Effects of high-calorie diet on puberty onset in female rhesus monkeys. Endocrinology 153:1696-1705.

Uguz C, Varisli O, Agca C, Agca Y (2009) Effects of nonylphenol on motility and subcellular elements of epididymal rat sperm. Reprod Toxicol 28:542-549. 
